ISSN: 2386-3919 - e-ISSN: 2386-3927

DOI: https://doi.org/10.14201/et20173516579

\title{
ANÁLISIS CIENTÍFICO DESCRIPTIVO: PROGRESO DEL SISTEMA EDUCATIVO DE ARABIA SAUDITA
}

\section{Descriptive scientific analysis: progress of the educational system of Saudi Arabia}

\author{
A. M. PROFANTER \\ Correo-e: annemarie.profanter@unibz.it
}

Recibido: 09/09/2016; Aceptado: 08/03/2017; Publicado: 31/05/2017

Ref. Bibl. A. M. PROFANTER. Análisis científico descriptivo: progreso del sistema educativo de Arabia Saudita. Enseñanza \& Teaching, 35, 1-2017, 65-79.

RESUMEN: Arabia Saudita tiene como objetivo el desarrollo de una sociedad basada en el conocimiento. Los cambios demográficos y el crecimiento económico que han ocurrido en poco tiempo en Arabia Saudita provocaron cambios radicales en el sistema educativo. Las instituciones de educación superior están en expansión y la colaboración internacional se ha intensificado. La política de segregación de género, que se basa en una sociedad neopatriarcal favorecida por el sistema tribal, es un importante elemento cultural de la sociedad saudita e influye en su cultura educativa.

Este artículo presenta una descripción científica y analiza los principales elementos del sistema educativo superior saudita utilizando los pocos datos disponibles, ya que el acceso a las estadísticas oficiales es limitado. El estudio de la Universidad Prince Mohammad Bin Fahd está basado en la experiencia de la autora, quien enseñó allí como profesora visitante durante los años académicos 2006 y 2008. Al ser el primer instituto privado en admitir estudiantes de ambos sexos tuvo que hacer frente a muchos desafíos. Además, el impacto de colaboraciones internacionales se identifica por medio del análisis del «Programa Escolar King Abdullah», el cual proporciona a miles de estudiantes la oportunidad de estudiar en el extranjero.

Siempre y cuando la educación tenga una función global cumple también con una función nacional. Por lo tanto, la colaboración con universidades del Oeste ha creado en el reino desafíos para las recientes generaciones, equilibrando los valores occidentales impuestos a lo largo de su educación superior con su cultura tradicional. 
Debido a la política de segregación de género, el sistema educativo saudita representa diferentes obstáculos y oportunidades en particular para las estudiantes.

Palabras clave: Educación superior; Arabia Saudita; educación femenina; Programa Escolar King Abdullah; Universidad Prince Mohammad Bin Fahd.

SUMMARY: Saudi Arabia has set itself the goal of developing a knowledge-based society. Demographic changes and economic growth within short time in the Kingdom of Saudi Arabia led to radical changes of the educational system. Higher education institutions are in expansion and international collaborations are being intensified. The policy of gender segregation based on a neopatriarchal society favored by the tribal system is an important cultural element of Saudi society and influences the Saudi educational culture.

This article provides a scientific description and analyzes the main elements of the Saudi higher education system using the few data available due to the limited release of official statistics. Prince Mohammad Bin Fahd University is analyzed as a case study based on the author's experience who taught there as an academic years of 2006 and 2008. Being the first private institution to admit both male and female students it had to face several challenges. Furthermore, the impact of international collaborations is identified by exploring the "King Abdullah Scholarship Programme» which gives thousands of students the opportunity to study abroad.

Education while having a global function also fulfills a national function. Therefore, collaborations with Western universities in the Kingdom have created challenges for the recent generations in balancing Western values imposed throughout their higher education with their traditional culture. Due to the policy of gender segregation, the Saudi educational system represents different obstructions and opportunities particularly for female students.

Key words: Higher education; Saudi Arabia; female education; King Abdullah Scholarship Programme; Prince Mohammad Bin Fahd University.

\section{INTRODUCCIÓN}

El sistema educativo de Arabia Saudita ha tenido en los últimos años cambios notables. Las instituciones de formación no han crecido sólo a nivel nacional, también se han intensificado los programas de estudio a nivel internacional y la colaboración. El número de oportunidades educativas para los estudiantes está en expansión más allá de las fronteras del país. A pesar de que persisten las restricciones sociales y religiosas, las mujeres, en gran parte, están incluidas en estas oportunidades educativas, aunque encuentran límites en la elección de su itinerario educativo. Hay que tener en cuenta que las instituciones femeninas todavía no han logrado el mismo nivel cualitativo que las masculinas. Además, las oportunidades de trabajo para las mujeres son limitadas y en general se ve un paro juvenil elevado, porque los estudiantes no poseen los conocimientos requeridos por la economía. 
En este artículo se pretende ofrecer una breve visión general de los progresos logrados y enumerar las problemáticas persistentes. Empezando por el plan de desarrollo del Gobierno, se llega a ejemplos concretos de universidades fundadas en los últimos años que hacen visible el crecimiento de las instituciones de formación superior. Se describe la cultura educativa que se basa en la tradición de la segregación y se introduce el rol de la mutawwa'in, la policía religiosa. Para comprender el poder de esta última se presenta un ejemplo concreto de un hecho que sucedió en la Prince Mohammad University en la provincia oriental de Arabia Saudita. Además de estos compromisos a nivel nacional, se describirá un programa que se desarrollará para sostener la formación de jóvenes sauditas a nivel internacional. Un análisis cuantitativo del impacto del King Abdullah Scholarship Program demuestra que la mayor parte de los estudiantes sauditas en el extranjero estudian en universidades anglófonas y que hay un incremento notable de estudiantes dispuestos a aprovechar esta oportunidad. A pesar de todos los esfuerzos del Gobierno es difícil para las jóvenes la integración en el mundo del trabajo y las instituciones educativas tendrán que encontrar respuestas con cierta prontitud.

\section{EVOLUCIÓN DE LA FORMACIÓN UNIVERSITARIA}

En los años cincuenta solo una elite podía beneficiarse de las oportunidades educativas, mientras que en el 2009 el 86,1\% de la población saudita está alfabetizada (United Nations Development Program, 2012). La primera y propiamente dicha universidad, King Saud Bin Abdul Aziz University, abrió sus puertas al público en 1957. "Since 1957 when modern university education began with a single institution with twenty-one students and a staff of nine it has grown until twenty-five years later in 1982 higher education had grown to seven institutions with 63,563 students and a teaching staff of 6,906» (Saleh, 1986: 17).

Bajo la guía del rey Abdullah bin Abdulaziz al Saud se tomó la decisión de utilizar la riqueza de Arabia Saudita para sostener el desarrollo del conocimiento de la población creciente. Según el Arab Human Development Report (2011) de los 80 al 2011 la población saudita ha aumentado el 286,5\%. En el 2013 la población ha llegado casi a los 30 millones de habitantes, con una media de edad de 26 años (wKÖ, 2014). En el 2012 la mayor parte (67,4\%) de la población estaba entre los 15 y los 64 años, el 29,7\% por debajo de los 15 años y solamente el 2,9\% por encima de los 64 años (wKÖ, 2014). Desde el 2000 el crecimiento de la población es más lento: del 2,6\% al 1,9\% en el 2012 (wKÖ, 2014).

El plan de desarrollo quinquenal Ninth Five-Year Development Plan (20102014) prevé que el 50,6\% del presupuesto será utilizado para el desarrollo de los recursos humanos, ya sea para la formación inicial o para la formación continua. También esto ha sido un paso importante para lograr el objetivo del reino en el desarrollo de una sociedad basada en el conocimiento. Desde la toma de poder del rey Abdullah han surgido 52 universidades, de las cuales 24 son públicas (46\%), 8 son privadas (15\%) y 20 son colegios privados (39\%) (Denman y Hilal, 2011). 
Según el Ministerio de Instrucción Superior (2009), se van a construir una serie de nuevos edificios, de los cuales 25 serán escuelas para el desarrollo tecnológico, 28 institutos técnicos y 50 institutos de formación industrial. Además, el Gobierno trata de ampliar y diversificar los programas postgrado que se ofrecen en el reino, tratando de aumentar la cantidad de estudiantes que los frecuentan hasta el 5\%. Además, este plan promueve la innovación en la ciencia y la tecnología, proporcionando cada año 240 millones de dólares de becas de estudio para proyectos de investigación. Otras iniciativas comprenden la creación de 10 centros de investigación, 15 centros de innovación tecnológica universitaria en colaboración con la ciudad King Abdullah City for Science and Technology (KACST) y otras 8 incubadoras tecnológicas para la KACST y otras universidades. El Gobierno continuará además promoviendo la colaboración entre universidades y compañías internacionales.

En Medio Oriente, en general, se trata de sostener de la misma manera la preparación internacional de los estudiantes, así como reforzar la investigación. En América del Norte y en América Latina, en comparación con Europa, dan más importancia a la preparación internacional de sus propios estudiantes. Las universidades africanas, por su parte, dan más importancia a la investigación (Al-Ohali y Burdon, 2013). En el caso de Arabia Saudita no hay que olvidar las inversiones del Gobierno destinadas a miles de estudiantes enviados al extranjero para realizar estudios universitarios y en especialidades necesarias para el crecimiento y el desarrollo de los recursos de este país.

Durante años, hubo pocas universidades estatales que solamente podían atender a una minoría, actualmente el número de universidades privadas ha aumentado y de esta manera se está contribuyendo a alcanzar el número de instituciones educativas necesario. Un ejemplo es la Prince Mohammad Bin Fahd University, abierta en el 2006, que fue la primera en la provincia oriental de Arabia Saudita en ofrecer puestos de estudio para estudiantes de sexo masculino y femenino en estructuras coeducativas (aunque en realidad se trata de estructuras separadas, como se discutirá en seguida). Los colegios de dimensiones más pequeñas, que antes estaban conectados a universidades más grandes, se están separando para formar nuevas universidades promovidas y financiadas por el Gobierno.

Por esto, una institución como la King Abdullab University for Science and Technology (KAUST) se ha desarrollado como institución de investigación sobre el arte, situada en las cercanías de Jeddah, que además en el 2009 rompió con la tradición de la segregación. De la misma manera, el centro de investigación para la salud Prince Naïf Center for Health Science Research fue desarrollado por la King Saud University de Riyadh, concentrándose en la educación de la propia población.

Además de este desarrollo, también se ha incrementado la formación de las mujeres, por ejemplo, la Princess Noura University al norte de Riyadh, abierta nuevamente en el 2011. El complejo, que cubre más de ocho millones de metros cuadrados, ha costado aproximadamente 11.500 millones de dólares. Alberga 15 
colegios y ofrece 50.000 puestos de estudio para mujeres, siendo así la universidad femenina más grande del mundo (Princess Noura University, 2014).

Además la Knowledge Economic City (KEC), que planea su inauguración para el 2020 en Madinah, se concentrará en la conexión entre la formación y la industria basada en el conocimiento y focalizará la formación en el campo médico. Se prevé que una vez completado KEC ofrecerá trabajo a más de 20.000 personas (Reisberg, 2011).

Estos son solamente algunos ejemplos de los cambios que se hacen actualmente en la promoción de la formación. Obviamente este incremento y las publicaciones científicas que las acompañan no pasan desapercibidos para la comunidad científica internacional. Un estudio publicado en el 2010 por la Royal Society of London sitúa a Arabia Saudita en un alto nivel de productividad científica, o sea que las publicaciones de investigación en revistas de impacto están entre los primeros lugares de la clasificación de los Estados del Golfo y en segundo lugar entre los Estados árabes. Sin embargo, estas investigaciones son frecuentemente efectuadas por científicos no sauditas, atraídos por los altísimos sueldos. Por este motivo, Bhattacharjee (2011) critica a Arabia Saudita por "comprar» científicos del extranjero con el fin de entrar en las clasificaciones de los centros científicos mundiales. En 2006 las universidades sauditas habían alcanzado posiciones escasas en las clasificaciones. Robertson, ya citado en el mismo articulo, justifica este comportamiento llamándolo un simple acto de capitalismo: «they have the capital and they want to build something out of it» (Bhattacharjee, 2011).

Los esfuerzos han aumentado la apreciación internacional y así Arabia Saudita se clasificó en el lugar 31 a nivel global por la eficacia del sistema de instrucción superior (Ministerio de Instrucción Superior, 2009). Tal inversión podría resultar minúscula en comparación a los beneficios finales de desarrollo de los recursos industriales, comerciales y económicos. Tanto las instituciones estatales como las privadas se han empeñado en entrar en las clasificaciones de las mejores universidades del mundo. Así, en el 2010 el Webometrics of World Universities ha clasificado 3 universidades sauditas entre las primeras 200 a nivel mundial y otras 6 universidades fueron incluidas en la lista de las mejores 10 universidades en los Estados del Golfo, del mundo árabe y en los países islámicos. En el 2009 la King Saud University fue la única admitida en la clasificación académica de las universidades mundiales, conocida como ranking de Shangai, entre las primeras 500 universidades internacionales (Ministerio de Instrucción Superior, 2009). Los ambiciosos esfuerzos de Arabia Saudita demuestran resultados positivos a pesar de que los puntos débiles de los sistemas de clasificación internacional sean evidentes y en el caso de los países del Golfo discutidos con referencia a sus relaciones comerciales con las mejores universidades de Europa y de Estados Unidos y el intercambio de profesores. 


\section{Culturas EDUCATIVAS. CONCEPTOS DivERGENTES}

La política de segregación de género basada en una sociedad neopatriarcal favorita del sistema tribal es un elemento cultural fundamental de la sociedad saudita. A esto se agrega la religión nacional del islam que influencia cada aspecto de la vida y, a veces, no puede separarse de lo que nosotros llamaríamos aspectos "profanos» de la vida. Junto a todo esto están los conceptos de Wasta y la importancia del mutawwa'in (policía religiosa). El concepto de Wasta influencia todos los aspectos de la sociedad y se refiere a los contactos personales o del poder de la realización de las propias intenciones, por ejemplo, también a nivel administrativo. A finales de los años 70 y 80, la mutawwa'in, a causa de un desconcierto social causado por ella misma, generó un poder y una autoridad notables, que todavía hoy ejerce a diferentes niveles. Mientras que la cultura de Oriente Medio tiende a tener muchos elementos comunes, Arabia Saudita presenta una interpretación mucho más fuerte o "pura" de elementos religiosos-culturales.

La educación, aun teniendo una función global, tiene también una función de cohesión nacional. La educación occidental se percibe como liberal por la mayor parte de los árabes, por tanto, se aprecia como sospechosa por muchos islamistas. «[...] many Muslims are Islamist and almost no very pious Muslims are liberals, most Muslims remain conservative, traditional believers. This group includes the majority of clerics and ordinary people» (Rubin, 2006: 101). Esto significa que, según Rubin, muchos musulmanes son islamistas y son conservadores y creyentes tradicionales. Otros retos para el crecimiento de las instituciones educativas son las restricciones religiosas y las costumbres conservadoras. Los institutos de instrucción superior modernos fueron rodeados de una sociedad musulmana conservadora, que contiene muchos retos como, por ejemplo, la infrautilización del potencial femenino para el desarrollo social.

La policía religiosa es particularmente influyente en el reino de Arabia Saudita, donde se practica la forma conservadora del islam del Wabhabi. Arabia Saudita, en el período después de la nacionalización, se concentró en la ideología del islam de Wahhabi que se basa en la filosofía de género, que puso énfasis durante la primera mitad del siglo xx junto con la conquista de Ibn Saud (Doumato, 2000: 42). La familia Al Saud, en colaboración con los religiosos wahbabiti, unió las regiones de la península árabe superior a lo que hoy es conocido como el reino de Arabia Saudita. Esta unificación ha generado una enorme expansión de los aspectos burocráticos que sostienen las infraestructuras del país y la actuación de los reglamentos reales y de la Shari'a.

Occidente frecuentemente ve a la familia Al Saud como extremadamente abierta y liberal en la interpretación del islam en un contexto moderno. Pero es importante no olvidar que el país se compone de diferentes fracciones que están ligadas principalmente a través de un enlace y de acuerdos mantenidos entre los Al Saud y el clero de Wabhabi. El rey Abdullah no tiene poder ilimitado, sino que tiene que respetar los sentimientos de la familia reinante, así como los del estatuto religioso. 
El pertenecer a la religión del islam es un factor determinante para garantizar puestos de trabajo y la admisión a las instituciones educativas, aunque no sea un criterio aplicado oficialmente. Un ejemplo relevante que influye en la educación es la asunción de personal y la admisión de los estudiantes con referencia a sus apellidos y afiliaciones tribales. Por escrito no hay alguna discriminación basada en la pertenencia religiosa; sin embargo, en la práctica se trata de un factor muy importante que nunca se discute ni admite abiertamente.

La segregación de los sexos en la instituciones educativas es realizada de manera diferente según la legislación nacional y religiosa. Antes que nada, las instituciones educativas en Arabia Saudita son establecidas principalmente para los hombres. Todos los itinerarios de estudio están abiertos para el género masculino; sin embargo, las instituciones creadas principalmente para el sexo femenino ofrecen estudios tradicionales, "específicamente femeninos", como medicina y diseño de interiores. Otras instituciones, como KFUPM, disponen de colegios separados dentro de la universidad para las mujeres.

Sin embargo, el desarrollo hacia una sociedad basada sobre el conocimiento es una empresa a largo plazo. El país ha dado muchos pasos para invertir en su capacidad de innovación. El reino está realizando fuertes inversiones para su sistema educativo, pero esto no se refleja necesariamente en su calidad. Pedagógicamente las lecciones presenciales y la memorización de informaciones (rote learning) asumen todavía un papel fundamental, de esa manera, los output creativos son el punto débil de Arabia Saudita. Muchos profesores universitarios empezaron su función de enseñantes sin ninguna formación pedagógica. Expertos en su ámbito son seleccionados según sus títulos. Como consecuencia, enseñan y examinan de la misma manera de como les fue enseñado, con lecciones presenciales y transmitiendo las informaciones que los alumnos repiten en los exámenes (Al-Ghamdi y Tight, 2013).

El sistema educativo está fuertemente influenciado por la cultura. En el caso en que se propongan alternativas para el sistema, estas vienen muchas veces consideradas como «buenas ideas» tomadas de la literatura y prácticas internacionales (Smith y Abouammoh, 2013a). El estilo occidental de escolarización se ve como el difusor de la doctrina del capitalismo, del gobierno centralizado, de la sociedad basada en los méritos y la idea de progreso (Leach, 1994). Por lo tanto, la rápida expansión de las universidades afiliadas americanas, británicas y europeas, con sus facultades eurocéntricas, ha creado un reto para las recientes generaciones sauditas: encontrar un equilibrio para superar los cuadros occidentales, impuestos por las autoridades, como se profundizará en el apartado siguiente.

\section{ANÁlisis DE Un EJEMPlO: EL CUADRO TRANSNACIONAL DE PMU}

El concepto de la Prince Mohammad University (PMU) en la provincia oriental de Arabia Saudita ha sido promovido por un grupo de 52 hombres empresarios sauditas bajo los auspicios del príncipe Mohammad Bin Fahad, gobernador de la 
provincia oriental que donó el terreno para este proyecto. El objetivo era impartir formación a los estudiantes de ambos sexos. Además de satisfacer las exigencias de la economía en crecimiento, querían desarrollar un instituto donde las estudiantes pudieran seguir viviendo en su hogar y a la vez recibir una formación occidental. Esta formación occidental fue diseñada por un grupo de 22 universidades texanas junto a la asociación Texas International Education Consortium (TIEC).

La Prince Mohammad University fue la primera institución privada en la provincia oriental de Arabia Saudita accesible para hombres y mujeres. Por lo tanto, el concepto de institutos de coeducación occidentales se diferencia mucho del de Arabia Saudita. "PMU admits both male and female students; however, the campus is divided into areas that maintain gender separation». Como resultado, aunque asistan a la misma facultad, los géneros se mantienen segregados en campus diferentes. Además, hay facultades en las que son admitidas solo mujeres, como, por ejemplo, la Facultad de Diseño de interiores (PMU, 2014). Además, cada colegio tiene a su presidente, que generalmente es de sexo masculino y mantiene la primera posición. El cargo de la subdirección está ocupada por mujeres.

Una universidad privada como la PMU, creada inicialmente como instituto coeducativo, funciona de manera muy diferente a lo que los occidentales se esperarían. En una entrevista al decano académico, contó un incidente ocurrido el primer día en la universidad PMU abierta en septiembre 2006: "Los estudiantes de sexo masculino y femenino estaban en la misma construcción y separados en lados opuestos del edificio. El personal docente y administrativo fue autorizado a reunirse en una zona central. Sin embargo, esto fue visto por la "Muttawa" que había ido a ver qué pasaba en el campus". Lo sucedido fue transmitido al gobernador, diciendo que esto no estaba permitido. El artículo 155 de la Policy Education de Arabia Saudita exige una separación rigurosa de hombres y mujeres en todos los niveles de formación con cuatro excepciones: jardín la infancia, jardín de niños, algunas escuelas primarias privadas y universidades médicas. El ejemplo de la PMU demuestra la influencia de las normas socio-religiosas y las dificultades en la aplicación de estándares educativos occidentales.

\section{King AbDullah Scholarship Programme}

En el 2005 surgió el programa de becas King Abdullah, que patrocina a estudiantes sauditas altamente cualificados, ofreciéndoles la oportunidad de estudiar en el extranjero. El programa incluye cursos de licenciatura y cursos de doctorado bajo la supervisión del Ministerio de Instrucción Universitaria. Una vez admitidos al programa, los estudiantes pueden escoger estudiar en 24 países diferentes (Smith y Abouammoh, 2013a). Arabia Saudita tiene el porcentaje más alto en el mundo de estudiantes becados por el Gobierno. En el 2013, el programa ha sido prorrogado hasta el año 2017 (Pavan, 2013).

Los datos del Ministerio de Instrucción Universitaria afirman que la oportunidad de estudiar en el extranjero atrae a muchos estudiantes sauditas. El número 
total de estudiantes inscritos en universidades fuera de Arabia Saudita ha crecido un 509\% entre el 2006 y el 2010 (14.523 en el 2006 y 88.435 en el 2010). En el 2013 el número total de los estudiantes sauditas becados por el Gobierno para estudiar en el extranjero ha aumentado llegando a 120.000 (Smith y Abouammoh, 2013a). Así, Arabia Saudita se encuentra en el tercer lugar de la escala mundial en referencia al número de estudiantes que estudian en el extranjero después de China e India (Pavan, 2013).

En el 2010, los estudiantes se inscribieron sobre todo en América del Norte (38.086), en Europa (19.573) y en Medio Oriente (10.600). La mayor parte de los estudiantes admitidos en el programa elige estudiar en países anglófonos, sobre todo, en los Estados Unidos (27.688 en el 2010), en el Reino Unido (16.552 en el 2010) y en Canadá (10.398 en el 2010). También Egipto parece ser una meta apreciada por los estudiantes sauditas (7.250 en el 2010). Un motivo podría ser que la instrucción universitaria se da preferentemente en lengua inglesa y que se trata de todos modos de un país árabe e islámico. En los países árabes, la mayor parte de los estudiantes se inscribe en las universidades situadas en Jordania (5.213 en el 2010).

A partir del 2006 hasta el 2010 el número de estudiantes en el extranjero ha aumentado en Australia y Nueva Zelanda. De 577 a 9.999 estudiantes en números absolutos, que corresponden a un aumento del 1.633\%. Una imagen semejante se muestra en Europa, donde en este período el número de estudiantes sauditas ha aumentado un $1.271 \%$ (en números absolutos de 1.428 a 19.573). Igualmente, en África del Norte el número de estudiantes sauditas ha aumentado un 1.159\% (de 599 a 7.544). En comparación al número de estudiantes de Norteamérica (comprende los Estados Unidos y Canadá), parece haber aumentado solo ligeramente, pero todavía con un porcentaje notable del 293\% (de 9.693 a 38.086) (Denman y Hilal, 2011).

Recientemente, muchos estudiantes sauditas han manifestado dudas acerca de la clasificación mundial publicada por su universidad huésped, en cuanto que hay una mayor conciencia y preocupación sobre lo que constituye la calidad de la educación, incluso en un programa patrocinado en el extranjero. La mayoría de los estudiantes afirma que la razón que los ha impulsado a estudiar en el extranjero es la esperanza de mejorar sus posibilidades en el mundo laboral o la oportunidad de continuar sus estudios universitarios. Sin embargo, afirman que tienen la intención de regresar a Arabia Saudita después de los estudios (Bukhari y Denman, 2013).

La mayor parte de los estudiantes sauditas en el extranjero están becados por el Gobierno, pero hay algunos casos raros en los cuales se autofinancian. La beca de King Abdullah representa un programa que crea una nueva generación de estudiantes sauditas con experiencias interculturales. La transferencia de conocimiento más allá de las fronteras geográficas se ve como un método eficaz para aumentar la productividad y creatividad de las fuerzas laborales. Las empresas sostienen este apoyo por parte del Gobierno, porque tienen necesidad de empleados cualificados. Además, se espera que este programa influya internamente y externamente también en otros aspectos sociales (Pavan, 2013). 
Algunos académicos no sauditas consideran que el objetivo final de este programa es la difusión del islam a nivel internacional. El fin declarado del programa es generar fuerzas laborales competitivas a nivel internacional (Bukhari y Denman, 2013). Un estudio de Hilal (2011) sugiere que un importante resultado del programa es la expansión de la visión del mundo de los estudiantes sauditas y de darles la oportunidad de entrar en contacto con otras culturas. Los estudiantes sauditas que estudian en el extranjero están ayudando a personas en los países occidentales a comprender las costumbres y tradiciones sauditas y son apreciados por su hospitalidad y su sed de conocimiento (Bukhari y Denman, 2013).

\section{LA EDUCACIÓN FEMENINA}

El rey Abdullah y la familia reinante de Al-Saud quieren demostrar que están dispuestos a hacer entrar a las mujeres en "todo tipo de ambiente» que pudiera interesarles (Wall Street Journal, 2007). Sin embargo, lo que el Ministerio ha decretado y los tribunales de la Shari'a salvaguardan puede diferenciarse de las dinámicas de las estructuras de poder local tradicional. Dentro del sector educativo existen debates contrastantes. El rey puso a disposición un presupuesto notable para becas de estudio internacional para las mujeres (Reisberg, 2011). A causa de las disposiciones Mahram y la necesidad de un acuerdo de un pariente masculino para la búsqueda de un trabajo, instrucción o viaje («a male relatives agreement before seeking work, education or travel») (Vidyasagar y Rea, 2004: 266), estos fondos no son frecuentemente utilizados por el público porque las familias no tienen la posibilidad de pagar los costes a dos de sus miembros para que viajen al extranjero. El número de mujeres que estudian en el extranjero ha aumentado en los últimos años alcanzando el 20\% (Bukhari y Denman, 2013).

Un paso importante -también simbólico- dado por el rey Abdullah es el nombramiento de un ministro adjunto mujer en el 2005, la posición más alta que una mujer saudita haya alcanzado en el Gobierno (Associated Press, 2009, par. 3-4). Nura el Fajes, como secretaria adjunta de la Instrucción Pública, es la primera mujer en una posición de alta responsabilidad en el Gobierno. Desde el 2013 en la consulta llamada Majlis al-Shura, formada por 150 delegados elegidos por el rey, 30 puestos son ocupados por mujeres (Auswärtiges Amt, 2014). Además hay mujeres que trabajan como doctoras, profesoras y mujeres de negocios, además de las empleadas en los diferentes medios de comunicación y en las fábricas (Jamjoom y Kelly, 2013).

La segregación en las universidades de Arabia Saudita mantiene, sin embargo, la convicción de que la educación femenina no es totalmente apreciada y que las mujeres son subordinadas. Los parientes, en las zonas rurales, persiguen la idea de que es más importante garantizar a sus hijas un buen matrimonio que una independencia financiera. Por lo tanto, la educación de las mujeres es vista también como una posibilidad de aumentar las oportunidades de matrimonio (Jamjoom y Kelly, 2013). 
Además, no todas las disciplinas académicas del reino están actualmente abiertas para las mujeres. Según un informe publicado por el Wall Street Journal (2007), en el 2006 las mujeres fueron admitidas en la Facultad de Derecho por primera vez. Sin embargo, no hay ninguna garantía de que serán autorizadas a ejercer un trabajo en su país, pero podrían hacerlo en el reino de Bahrain. Actualmente, las oportunidades educativas para las mujeres, basadas en la educación americana y europea y adaptadas a un sistema segregativo, hacen que esta generación de mujeres estudiantes no pueda adaptarse a la realidad del mundo laboral donde podrían estar incluidos los hombres.

Es importante subrayar que el número de facultades femeninas ha aumentado un $242 \%$ en el período entre el 1990 y el 2004, mientras que el número de facultades masculinas ha aumentado un 152\%. Estos datos se reflejan también en el número de las inscripciones: ha aumentado el 512\%, en las mujeres y en los hombres el 339,2\%. En el 2013 el 60\% de los estudiantes resultan ser mujeres. En su lugar si se consideran los empleados de las universidades se nota un desequilibrio: casi dos tercios son hombres. En conclusión, existe una gran desproporción entre estudiantes mujeres y personal en las facultades femeninas en comparación a las masculinas (Jamjoom y Kelly, 2013).

Generalmente, encontrar una ocupación no es fácil para las mujeres, pero en los últimos años la situación ha cambiado: el Ministerio afirma que el 17\% de todos los investigadores sauditas son mujeres. De hecho, según un informe de la UNESCO titulado Mujeres en la ciencia: subrepresentadas y subestimadas (Women in science: under-represented and under-measured), resultó que el número es superior al de Alemania (12\%), Japón (12\%) y Corea (11\%). El mismo porcentaje de investigadores de sexo femenino (17\%) se encuentra en Luxemburgo. Según el Global Education Digest de la UNESCO (2009) son más numerosas las mujeres sauditas, en comparación a las occidentales, que se inscriben en la universidad y se gradúan. Esto indica un gran progreso para las mujeres del reino. Sin embargo, el porcentaje de mujeres empleadas con más de 15 años de servicio corresponde solamente al 21,2\% (United Nations Development Program, 2012).

En el 2005 se desarrolló una nueva ley con el fin de aumentar el empleo de las mujeres y de los habitantes en general: datos oficiales afirman que el 49\% (6,2 millones) de la fuerza laboral son no sauditas, el 14,7\% son mujeres sauditas y el restante $36,3 \%$ son hombres sauditas. La tasa de paro demuestra una gran discrepancia de genero: el 21,7\% de las mujeres y el 7,6\% de los hombres están parados (EIU Views Wire Middle East, 2005). Aunque estas cifras son desiguales, persiste la duda de que estos datos no representan la actual y real situación económica. En su libro Mujeres al poder: el debate árabe sobre las mujeres en el trabajo (Women Power: the Arab Debate on Women at Work) Hijab indica tres condiciones que tienen que cumplirse antes de que las mujeres puedan estar completamente integradas en el mundo laboral retribuido: necesidad, oportunidad y capacidad. Una nueva ley sobre el trabajo en Arabia Saudita proclama que "las mujeres tienen el derecho a trabajar en todos los campos que son apropiados a su naturaleza» (Middle 
A. M. PROFANTER

ANÁLISIS CIENTÍFICO DESCRIPTIVO: PROGRESO DEL SISTEMA EDUCATIVO DE ARABIA SAUDITA

East Monitor, 2005). La ambigüedad en esta declaración se refleja en la afirmación de Pharaon (2004: 358): según él, el papel clave de las mujeres es mantener a la familia. Ella es la madre que transmite las tradiciones culturales y religiosas, que refuerza la solidaridad y la lealtad hacia la familia. Un temor real, según él, es que, si las mujeres adoptan otros papeles, ponen en riesgo el sistema social interno.

\section{CONClusiones}

El crecimiento de la población, los cambios sociales, las normas culturales y la economía han influido en el desarrollo de las instituciones de formación en Arabia Saudita (Al-Dali, Fnais y Newbould, 2013). Para lograr el objetivo del Gobierno, es decir, el desarrollo de una economía del conocimiento, hay todavía muchas dificultades que superar. Persisten tensiones entre las viejas visiones académicas y las normas culturales. Un equilibrio adecuado entre estas dos hará posible crear un sistema de instrucción de calidad, manteniendo las tradiciones culturales basadas en la religión (Smith y Abouammoh, 2013b).

A nivel internacional las actividades de investigación y su impacto son todavía bajos respecto a los estándares internacionales y todavía no es posible decir que los esfuerzos del Gobierno para la producción de conocimiento tengan impactos positivos en la industria (Al-Ohali yShin, 2013).

Las mujeres representan una fuente intelectual que podría ayudar al desarrollo económico de Arabia Saudita. Han aumentado las posibilidades de acceso y de participación en las instituciones de formación para las mujeres, pero aún no han alcanzado el nivel cualitativo de las instituciones masculinas. Un paso hacia un mejoramiento sería dado asegurando las mismas posibilidades de acceso a las bibliotecas y a los medios de investigación on-line que tienen los hombres (Smith y Abouammoh, 2013b). Además, si se desean mantener los principios de la segregación y una buena calidad de las instituciones, será inevitable emplear más mujeres -también en las universidades- ya que son mayoría en inscribirse en las universidades con respecto a los hombres (Jamjoom y Kelly, 2013).

Una manera de eliminar los obstáculos culturales en Arabia Saudita está en el uso de la nueva tecnología del aprendizaje mixto. Seguramente hay desafíos que superar, como dar una formación adecuada a los estudiantes de modo que puedan sentirse seguros en el nuevo ambiente de aprendizaje y de sacar el máximo provecho de este, de todos modos esto parece ser un acercamiento prometedor.

Aunque los estudiantes estén sostenidos por el Gobierno, muchos abandonan los estudios, probablemente la causa es la falta de motivación (Bosbait y Wilson, 2005). La universidad es vista como un espacio protegido que responde a las exigencias de los jóvenes (Tétreault, 2011). Además, los jóvenes licenciados parecen estar escasamente preparados para el mundo laboral. Los mismos estudiantes afirman en una investigación que su formación no era apropiada para el mercado laboral. Solo el $27 \%$ de los parados consideraba su propia instrucción como útil (Bosbait y Wilson, 2005). De hecho, el 18\% escoge estudios en ámbitos de ciencias, 
matemática o ingeniería, mientras que el 40\% escoge estudios islámicos (Bosbait y Wilson, 2005). Así también Tétreault (2011) describe el fenómeno de cómo la formación de los estudiantes no corresponde a las exigencias del mercado del trabajo.

Esto conduce a un aumento del desempleo juvenil en Arabia Saudita que llega a alcanzar el 32\% (Pakkiasamy, 2004). Además, considerando solamente a la población laborable, dos tercios de ella son del extranjero. En el sector privado hasta el 95\% de los trabajadores son extranjeros (Pakkiasamy, 2004). En el 2002 el «Human Resources Development Fund" planea abastecer el 75\% del pago de los salarios a los trabajadores sauditas empleados por el sector privado durante la formación profesional (Bosbait y Wilson, 2005). Los intentos del Gobierno de sustituir a los trabajadores extranjeros con sauditas, por ejemplo en trabajos como taxistas, en el comercio de tiendas de alimentos y en general en los ámbitos de trabajo físico, resultaron ser un propósito lleno de problemas, porque parecen poco atractivos a los sauditas debido a los salarios relativamente bajos y al estatus que estos tienen. Así la solución parece ser el aumento de los puestos de trabajo en el sector de los servicios (Bosbait y Wilson, 2005). Desde el 2000 el Gobierno de Arabia Saudita se ha empeñado en aumentar la calidad de vida de la propia población. Las iniciativas conciernen a los servicios sanitarios, la financiación de las fuerzas militares y en particular la educación, que es vista como una necesidad y como la solución para asegurar a las fuerzas laborales una posición competitiva a nivel global (Denman y Hilal, 2011).

Este artículo referencia las descripciones científicas y los análisis de los elementos principales del sistema de instrucción superior saudita con los pocos datos disponibles debido a la publicación limitada de las estadísticas oficiales. Hay retos que enfrentar en cuanto a la calidad de la instrucción y el elevado número del paro. Sin embargo, el rey ha cumplido con su deber con el fin de diversificar la economía. Ahora podemos ver solo los resultados limitados de esta política, pero esperamos que, en el futuro, la familia reinante desarrolle plenamente el concepto de una economía basada en el conocimiento.

\section{REFERENCIAS BIBLIOGRÁFICAS}

Al-Dali, W.; Fnais, M. y Newbould, I. (2013). Private Higher Education in the kingdom of Saudi Arabia: Reality, Challenges and Aspirations. En L. Smith y A. Abouammoh (Eds.). Higher Education in Saudi Arabia (pp. 127-136). Dordrecht: Springer Science +Business Media.

Al-Ghambi, S. y Tight, S. (2013). Selecting and Developing High-Quality Academic Staff. En L. Smith y A. Abouammoh (Eds.). Higher Education in Saudi Arabia (pp. 83-93). Dordrecht: Springer Science +Business Media.

Al-Ohali, M. y Burdon, S. (2013). International Collaboration. En L. Smith y A. Abouammoh (Eds.). Higher Education in Saudi Arabia (pp. 159-166). Dordrecht: Springer Science +Business Media. 
A. M. PROFANTER

ANÁLISIS CIENTÍFICO DESCRIPTIVO: PROGRESO DEL SISTEMA EDUCATIVO DE ARABIA SAUDITA

Auswärtiges Amt (2014). Innenpolitik. Descargado el 3 de julio de 2014. http://www. auswaertiges-amt.de/DE/Aussenpolitik/Laender/Laenderinfos/SaudiArabien/Innenpolitik_node.html.

Bhattacharjee, Y. (2011). Saudi Universities Offer Chas In Exchange for Academic Prestige. Science, 334, 1344-1345.

Bosbait, M. y Wilson, R. (2005). Education, School to Work Transitions and Unemployment in Saudi Arabia. Middle Eastern Studies, 41 (4), 533-545.

Bukhari, F. y Denman, B. (2013). Student Scholarships in Saudi Arabia: Implications and Opportunities for Overseas Engagement. En L. Smith y A. Abouammoh (Eds.). Higher Education in Saudi Arabia (pp. 1-12). Dordrecht: Springer Science +Business Media.

Denman, B. D. y Hilal, K. T. (2011). From barriers to bridges: An investigation on Saudi student mobility (2006-2009). International Review of Education, 57 (3-4), 299-318.

Doumato, E. A. (2000). Getting God's Ear: women, Islam, and healing in Saudi Arabia and the Gulf. New York: Columbia University Press.

EIU Views Wire Middle East (2005). Middle East/Africa. Saudi Arabia. Jobs for the girls? Country Monitor, 13 (35), 8.

Hilal, K. (2011). The impact of international higher education scholarships in Saudi Arabia. $\mathrm{PhD}$, University of New England, Armidale.

Jamjoom, F. B. y Kelly, P. (2013). Higher Education for Woman in the Kingdom of Saudi Arabia. En L. Smith y A. Abouammoh (Eds.). Higher Education in Saudi Arabia (pp. 117-125). Dordrecht: Springer Science +Business Media.

Leach, F. (1994). Expatriates as Agents of Cross-cultural Transmission. Journal of Comparative Education, 24 (3), 217-231.

Middle East Monitor (2005). Working Women. Middle East Monitor: The Gulf, 15 (12), 3-4.

Ministry of Higher Education (2009). Saudi's higher education achievements \& challenges. Global assessment \& international experts'views. Consultado el 25 de enero de 2012 de la base de datos de //www.mohe.gov.sa/en/default.aspx.

Pakkiasamy, D. (2004). Saudi Arabia's Plan for Changing Its Workforce. Descargado el 3 de julio de 2014. http://www.migrationpolicy.org/article/saudi-arabias-plan-changingits-workforce.

Pavan, A. (2013). A New Perspective on the Quest for Education: The Saudi Arabian Way to Knowledge Society. Higher Education Studies, 3 (6), 25-34.

Pharaon, N. A. (2004). Saudi Women and the Muslim State in the Twenty-First Century. Sex Roles, 51 (5/6), 349-366.

Prince Mohammad University (2014). Consultado el 3 de julio de 2014. http://www.pmu. edu.sa/About/Students.aspx.

Princess Noura Bint Abdul Rahman University (2014). Consultado el 3 de julio de 2014. http://www.pnuproject.com/.

Rubin, B. (2006). The Long War for Freedom. The Arab Struggle for Democracy in the Middle East. New Jersey: John Wiley \& Sons, Inc.

Saleh, M. S. (1986). Development of higher education in Saudi Arabia. Higher Education, 15 (1-2), 17-23. Consultado el 3 de julio de 2014. http://link.springer.com/article/ $10.1007 \% 2 \mathrm{FBF} 00138089$

Smith, L. y Abouammoh, A. (2013a). Higher Education in Saudi Arabia: Reforms, Challenges and Priorities. En L. Smith y A. Abouammoh (Eds.). Higher Education in Saudi Arabia (pp. 1-12). Dordrecht: Springer Science +Business Media. 
Smith, L. y Abouammoh, A. (2013b). Higher Education in Saudi Arabia: Conclusions. En L. Smith y A. Abouammoh (Eds.). Higher Education in Saudi Arabia (pp. 181-190). Dordrecht: Springer Science +Business Media.

United Nations Development Program (2012). Human Development Data for the Arab States. Saudi Arabia. Consultado el 3 de julio de 2014. http://www.arab-hdr.org/data/profiles/ SAU.aspx.

Vidyasagar, G. y Rea, D. M. (2004). Saudi women doctors: Gender and careers within Wahhabic Islam and a 'westernised' work culture. Women's Studies International Forum, 27 (3), 261-280.

Wall Street Journal (2007). Other Comments. Forbes, 180 (8), 24.

WKÖ - Witschaftskammer Österreich (2014). Länderreport Saudi-Arabien. Consultado el 3 de julio de 2014. http://wko.at/statistik/laenderprofile/lp-saudi-arabien.pdf. 